\title{
Model predictive control of HVDC power flow to improve transient stability in power systems
}

\author{
Y. Phulpin ${ }^{1}$, J. Hazra ${ }^{1}$, and D. Ernst ${ }^{2}$ \\ ${ }^{1}$ Department of Power and Energy Systems, SUPELEC, France (email@yphulpin.eu) \\ ${ }^{2}$ University of Liège, Belgium (dernst@ulg.ac.be)
}

\begin{abstract}
This paper addresses the problem of HVDC control using real-time information to avoid loss of synchronism phenomena in power systems. It proposes a discrete-time control strategy based on model predictive control, which solves at every time step an open-loop optimal-control problem using an $\mathrm{A}^{*}$ event-tree search. Different optimisation criteria based on transient stability indices are compared. The paper presents simulations results for two benchmark systems with 9 and 24 buses, respectively, and an embedded HVDC-link. The results show that the control strategy leads to a modulation of the HVDC power flow that improves significantly the system's ability to maintain synchronism in the aftermath of a large disturbance.
\end{abstract}

\section{INTRODUCTION}

High-voltage direct current (HVDC) technology has become widely used for power transmission over long distances through submarine or underground cable crossings. However, as an HVDC-link involves no coupling between voltage phase angles at the terminal buses, substituting AC lines by HVDClinks may disable natural damping properties of AC systems with respect to generator speed variations, which might consequently challenge the ability of the system to remain in synchronism [1]. To avoid those drawbacks, much research has investigated the use of the control settings offered by AC/DC converters based on power electronics to improve the system response in the face of sudden disturbances such as loss of load, generation, or transmission devices. Indeed, while driven by economic and technical objectives in the long-term, the settings for power flows through HVDC-links could be modulated during a short period of time to mitigate voltage angle fluctuations. In particular, several HVDC power flow modulation strategies have been proposed (see [2] and [3], for example) to increase operation margins with respect to loss of synchronism phenomena.

Loss of synchronism is defined in [4] as a particular type of instability that affects the rotor angle of one or several generators of an interconnected power system. It consists of increasing angle differences between interconnected generators, and generally results from a severe disturbance (e.g., short circuit on a transmission line) initiating an imbalance between the mechanical power received by a generator and its electrical power injection into the grid. The ability of the system to maintain synchronism in the aftermath of a contingency depends on the initial operating state, disturbance, and control actions. If the initial disturbance is not cleared within a certain time, some of the rotor angle differences exceed limit values related with the electromechanical coupling between generators, and the system loses synchronism. To avoid such phenomena, power system operators must schedule appropriate stability margins when tuning protection and operation devices.

To improve significantly the resilience of a system with HVDC-links with respect to loss of synchronism phenomena, we propose in this paper an approach based on model predictive control (MPC). As reported in [5], this technique has had a great success in control theory since the 1960s, and it has already been widely investigated for power system applications. For example, MPC is used in [6] to damp electromechanical oscillations with a variable reactance, in [7] for emergency voltage control, and in [8] to alleviate thermal overload. MPC is also proposed in [9] to control FACTS so as to make the system more robust to transient instability phenomena. To the best of the authors' knowledge, it is the first time MPC is applied to HVDC power flow modulation.

The MPC-based control scheme relies on real-time information collected through a wide-area monitoring system. It consists in computing at every discrete time instant a sequence of control actions that maximizes a transient stability index over a short time horizon and using the first action of this sequence as control setting for the HVDC-links. This approach is evaluated for different transient stability indices on two test systems with a single HVDC-link: a 3 machine 9 bus system and the IEEE 24 bus system. Under several restrictive assumptions, namely the state is fully observable, time delays are neglected, and the trajectory of the system can be accurately computed for any power flow modulation strategy, simulations show that the proposed technique allows a significant improvement of the system ability to remain in synchronism after critical events.

The paper is organized as follows. Section II describes the MPC methodology. Section III details how MPC can be suited to HVDC control to mitigate loss of synchronism phenomena. Section IV describes the power system model and presents the simulation results, and Section $\mathrm{V}$ concludes and proposes further research directions.

\section{GenerAl DESIGN OF THE MPC-BASED APPROACH}

Model predictive control is a decision-making technique that can be applied to time-variant finite-time control problems (i.e., the system properties may vary with time and the control focuses on a limited period of time). These problems are 
usually characterized by a discrete-time process, for which the dynamics $f: \mathbf{X} \times \mathbf{U} \times\{0,1, \ldots, N-1\} \rightarrow \mathbf{X}$ corresponds to the generic equation:

$$
\mathbf{x}[n+1]=f(\mathbf{x}[n], \mathbf{u}[n], n)
$$

where $N$ is the control horizon, $\mathbf{X}$ the space of system states, $\mathbf{U}$ the set of $P$ possible control actions, and $\mathbf{x}[n] \in \mathbf{X}$ and $\mathbf{u}[n] \in \mathbf{U}$ the system state and control action at time instant $n$, respectively.

The MPC strategy selects the control action to apply at instant $n$ by identifying a sequence of $H$ successive control actions that minimizes a cost function $C: \mathbf{X} \times \mathbf{U}^{H} \mapsto \mathbb{R}$ given the current state $\mathbf{x}[n]$. Then, it applies the first control action of this sequence and, at the next time step, reproduces the same process. The motivation for solving an optimal-control problem at every discrete instant, instead of only once at instant $n=0$ with $H=N$ and then applying the whole sequence of control actions, is twofold. First, by recomputing an open-loop sequence at every instant $n$, one may mitigate the sub-optimality problems related, among others, to the fact that Equ. (1) may not represent perfectly the dynamics of the real system and that the initial state may not be known exactly. Second, as the search space of the optimisation problem to be solved at every time step grows exponentially with the optimisation horizon, it is often better to work with $H$ much smaller than $N$.

To compute the optimal control sequence, one could make an exhaustive search over all the $P^{H}$ possible sequences of actions. However, even for relatively small values of $P$ and $H$, such a procedure is generally computationally too expensive. Several algorithms have thus been proposed in the literature (see [10] for example) to identify an optimal sequence of actions without having to evaluate every scenario. In an attempt to limit the computational burden associated with the identification of the optimal sequence of actions, we propose to use an algorithm introduced by [11], usually referred to by $\mathrm{A}^{*}$ algorithm. To compute $\mathbf{u}[n]$, this algorithm proceeds as follows.

- 1] Set $i=0, \mathbf{x}^{*}[0]=\mathbf{x}[n], C^{*}[0]=0$, set $S$ to the empty list \{\} , and $E=\{0, \mathbf{x}[n], 0,\{\}\}$ the list of explored nodes.

- 2] While $i<H$, do:

- 2a] Remove the first element of $E$.

- 2b] For every $p=1, \ldots, P$

* Compute $\mathbf{x}^{*, p}[i+1]=f\left(\mathbf{x}^{*}[i], \mathbf{u}^{p}[i], i\right)$.

* Compute $C^{*, p}[i+1]=C^{*}[i]+c\left(\mathbf{x}^{*, p}[i+1]\right)$.

* Add $\left[C^{*, p}[i+1], \mathbf{x}^{*, p}[i+1], i+1,\left\{S, \mathbf{u}^{p}[i]\right\}\right]$ to the set of explored nodes $E$.

- 2c] Order the list $E$ by increasing cost.

- 2d] Select the first element $\left[C^{\circ}, \mathbf{x}^{\circ}, i^{\circ}, S^{\circ}\right]$ in $E$.

- 2e] Set $i=i^{\circ}, \mathbf{x}^{*}[i]=\mathbf{x}^{\circ}, S=S^{\circ}$, and $C^{*}[i]=C^{\circ}$.

- 3] Set $\mathbf{u}[n]$ equal to the first control action of the list $S$.

\section{MPC-BASED HVDC CONTROL TO MITIGATE LOSS OF SYNCHRONISM PHENOMENA}

To apply the MPC-based approach to the HVDC power flow modulation problem, we have considered that the proposed strategy computes a new control variable $\mathbf{u}[n]$ at every discrete time instant $n \in \mathbb{N}$ in the aftermath of a disturbance initiated at instant $n=0$.

The vector $\mathbf{x}[n]$ gathers the values of the state variables at instant $n$, the control variable $\mathbf{u}[n]$ corresponds to the concatenation of the power flow $P_{k}^{D C}[n]$ through every HVDC-link $k \in\{1,2, \ldots, K\}$ at instant $n$. To reduce the set of possible values for $P_{k}^{D C}[n]$ and reduce computation times, the search space $\mathbf{U}$ is discretized and restricted to $P=M^{K}$ values (i.e., $M$ possible power flow settings by HVDC-link). The function $f$ corresponds to the power system dynamics. It is obtained from the integration of the continuous-time power system dynamics over time intervals of constant duration. At this stage, the control scheme relies on a real-time estimation of both the value of $\mathbf{x}[n]$ and a function $f$ that represents well the power system dynamics. Such an estimation is difficult, especially when a disturbance's location, effects on the postfault configuration, and duration have to be estimated. One way to achieve this in practice would be to leverage a real-time wide-area monitoring system that could eventually refresh the values of the state variable $\mathbf{x}[n]$ and the parameters of a predefined function $f$ approximating the dynamics of the system.

While different definitions of the cost function $C$ can be chosen, we will consider in this paper that $C$ corresponds to the sum of instantaneous costs $c(\mathbf{x}[n+1]), c(\mathbf{x}[n+2]), \ldots$, $c(\mathbf{x}[n+H])$, with $c(\mathbf{x})$ reflecting the degree of instability of the system in state $\mathbf{x}$. Hence, $C$ is defined as follows:

$$
C(\mathbf{x}[n], \mathbf{u}[n], \ldots, \mathbf{u}[n+H-1])=\sum_{i=n+1}^{n+H} c(\mathbf{x}[i]) .
$$

This definition of $C$ supposes that $n+H<N$. If this inequality does not hold true, the value of $H$ in the cost function (2) should be replaced by the largest value of $H$ that satisfies the above-written inequality.

The instantaneous cost function $c: \mathbf{X} \mapsto \mathbb{R}$, tested later in this paper, is defined by:

$c(\mathbf{x}[n])= \begin{cases}D(\mathbf{x})-D_{\min } & \text { if } \nexists i, j \in\left\{1, \ldots, N_{G}\right\} \text { such that } \\ & \left\|\delta_{i}[n]-\delta_{j}[n]\right\| \leq \delta_{\max } \\ c_{\text {pen }} & \text { otherwise }\end{cases}$

where $N_{G}$ represents the number of generators, $D(\mathbf{x})$ is a transient stability index, $D_{\min }$ its minimum value, and $c_{p e n}$ a large constant value that strongly penalizes system states outside of the domain of stability of the system ${ }^{1}$. In particular, this value should be chosen large enough to ensure that if there exists a sequence of actions that maintains the system inside its domain of stability, then a sequence of action that leads to

${ }^{1}$ In this paper, we will assume that the system has reached instability when the maximal angular deviation exceeds a limit value $\delta_{\max }$ chosen equal to $180^{\circ}$. 
instability is necessarily suboptimal. This can be achieved, for example, by choosing $c_{\text {pen }}=\left(\max _{x \in X} D(x)-D_{\text {min }}\right) \times H$, as it will be the case later in our simulations.

Three different transient stability indices $D: \mathbf{X} \mapsto \mathbb{R}$ will be used hereafter. They are referred to by $D_{P}, D_{C}$ and $D_{E}$ and correspond to transient stability indices introduced before in the literature (see, e.g., [12]). Those indices are based on rotor angle position, speed, and acceleration of every generator. The larger they are, the higher the degree of instability of the system. They are described hereafter.

1) The accelerating power index $D_{P}$ is defined in [12] by

$$
\begin{aligned}
& D_{P}(\mathbf{x}[n])= \\
& \sum_{i=1}^{N_{G}}\left(w_{i}[n]-w_{C O I}[n]\right)\left(\theta_{i}[n]-\theta_{C O I}[n]\right),
\end{aligned}
$$

where $w_{i}[n]$ and $\theta_{i}[n]$ represent the rotor speed and rotor angle of generator $i$ at instant $n$, respectively. $w_{C O I}[n]$ and $\theta_{C O I}[n]$ represent the rotor angle and rotor speed of the center of inertia of the system at instant $n$. They are computed as follows:

$$
\begin{aligned}
\theta_{C O I}[n] & =\frac{1}{M_{T}} \sum_{i=1}^{N_{G}} M_{i} \theta_{i}[n], \\
w_{C O I}[n] & =\frac{1}{M_{T}} \sum_{i=1}^{N_{G}} M_{i} w_{i}[n],
\end{aligned}
$$

where $M_{i}$ represents the inertia of generator $i$ and $M_{T}=$ $\sum_{i=1}^{N_{G}} M_{i}$ the overall inertia of the system.

2) The coherency index $D_{C}$ is defined in [12] by

$$
D_{C}(\mathbf{x}[n])=\sum_{i=1}^{N_{G}} g_{i}(\mathbf{x}[n])\left(w_{i}[n]-w_{C O I}[n]\right),
$$

with

$$
g_{i}(\mathbf{x}[n])=P_{m i}[n]-P_{i}[n]-\frac{M_{i}}{M_{T}} P_{C O I}[n],
$$

where $P_{m i}[n]$ is the mechanical power received by generator $i$ at instant $n, P_{i}[n]$ its electrical power output at instant $n$, and $P_{C O I}[n]=\sum_{i=1}^{N_{G}} P_{m i}[n]-P_{i}[n]$.

3) The energy index $D_{E}$ is defined as follows.

$$
D_{E}(\mathbf{x}[n])=\sum_{i=1}^{N_{G}}\left(w_{i}[n]-w_{C O I}[n]\right)^{2} .
$$

\section{EVAluation OF THE MPC-BASED APPROACH}

This section presents first the framework used to compare different HVDC power flow modulation strategies. Second, it details the power system model used in the simulations. Third, it reports simulation results.

\section{A. Analysis framework}

We detail in this subsection the test systems, simulation conditions, and some criteria to evaluate the effectiveness of the different variants of the MPC based strategy.

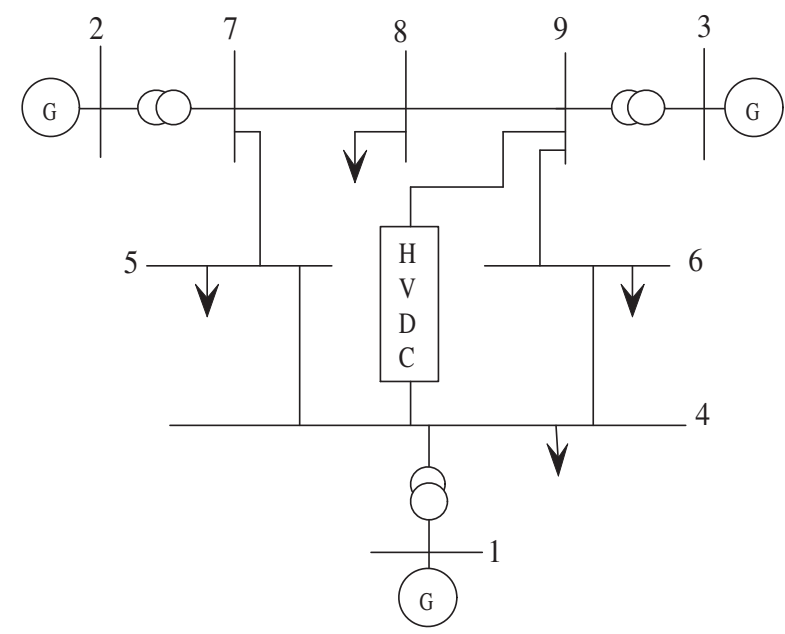

Fig. 1. 9 bus system with one embedded HVDC-link.

TABLE I

Pre-Fault bus Variables for the 9 Bus test System. $P_{i}$ And $Q_{i}$ REPRESENT REAL AND REACTIVE POWER INJECTIONS AT BUS $i . V_{i}$ AND $\delta_{i}$ REPRESENT THE VOLTAGE MAGNITUDE AND ANGLE AT BUS $i$, RESPECTIVELY.

\begin{tabular}{|c|c|c|c|c|}
\hline $\begin{array}{c}\text { Bus } \\
\#\end{array}$ & $\begin{array}{c}P_{i} \\
(\mathrm{MW})\end{array}$ & $\begin{array}{c}Q_{i} \\
\text { (MVAR) }\end{array}$ & $\begin{array}{c}V_{i} \\
\text { (p.u.) }\end{array}$ & $\begin{array}{c}\delta_{i} \\
(\text { deg.) }\end{array}$ \\
\hline \hline 1 & 72.07 & 71.42 & 1.040 & 0.00 \\
\hline 2 & 163.00 & 23.31 & 1.025 & 9.31 \\
\hline 3 & 185.00 & 40.69 & 1.025 & 8.08 \\
\hline 4 & -100.00 & -30.00 & 1.001 & -2.28 \\
\hline 5 & -125.00 & -50.00 & 0.974 & -4.16 \\
\hline 6 & -90.00 & -30.00 & 0.987 & -3.84 \\
\hline 7 & 0.00 & 0.00 & 1.015 & 3.70 \\
\hline 8 & -100.00 & -35.00 & 0.999 & 0.69 \\
\hline 9 & 0.00 & 0.00 & 1.007 & 2.05 \\
\hline
\end{tabular}

1) Benchmark systems : The proposed control strategy is applied to a 3 machine 9 bus system with one embedded HVDC-link. This system, depicted in Fig. 1, is initially operated under the pre-fault steady-state conditions described in Table I. The HVDC-link is connected to buses 4 and 9, and a $100 \mathrm{MW}$ power flow setting is applied during steadystate operation. It is considered that the link has a $200 \mathrm{MW}$ transmission capacity, and that the power flow setting has a resolution of $1 \mathrm{MW}$.

Further simulations were carried out on the IEEE 24 bus system presented in [13], where an HVDC-link is introduced between bus 21 and bus 11 . Under initial steady-state conditions, the HVDC-link voltage is $500 \mathrm{kV}$ at bus 11, and the incoming power flow at bus 21 is $400 \mathrm{MW}$. The transmission capacity of the HVDC-link is considered equal to $600 \mathrm{MW}$, and the power flow setting has a resolution of $5 \mathrm{MW}$.

2) Simulation conditions : In every simulation reported in this paper, we consider that the system is initially in steadystate conditions. At time $n=0$, a three phase to ground fault is applied to a bus. The simulation stops when the maximum simulation time, chosen equal to two seconds, is reached.

The faults are applied to the buses that are located next to 
the generators' transformers on the transmission network side. We have not considered contingencies on the HVDC-link in our simulations.

The control variables can only be refreshed at each discrete instant $n$ and the real-time between two successive discrete instants is 10 milliseconds. With such a simulation step, we have 200 as value for the optimisation horizon $N$.

3) Evaluation criteria: The time the system takes to reach instability after the occurrence of a contingency is referred to by time to instability (TTI).

We will report simulation results related to the MPC-based controller used with the three different stability indices $\left(D_{P}\right.$, $D_{C}$, and $D_{E}$ ) detailed in Section III. For every contingency and every instance of the MPC-based controllers, we will report whether the system is driven to instability according to the criterion defined here above. In such a case, we will also report the time to instability (TTI).

If for a given contingency, one controller drives the system to instability and another not, then this second controller is considered more effective. If two controllers drive the system to instability, we assume that the one that has the larger TTI is more effective, as it would allow more time for other emergency control measures (e.g., fast-valving, dynamic breaking) to steer away the system from instability.

The MPC-based strategies will be compared with three other control schemes for the HVDC-link. The first one (Optimal) selects a sequence of $N$ control actions that (i) avoids the system to reach instability if such a sequence indeed exists (ii) maximizes the TTI otherwise. This strategy can be considered optimal in terms of system response, provided that we are only interested in stability/instability diagnosis and TTIs. The second one corresponds to operating the HVDC-link with a constant current all the time $(C C)$, and the third one modulates the power flows by using a continuous-time PI control strategy $(P I)$ proposed in [2]. This latter strategy uses as input the voltage angles at the rectifier and inverter buses of the HVDClink. The proportional gain $K_{p}$ and the integral grain $K_{i}$ of the PI controller are chosen equal to 0.005 and 0.0001 , respectively, as in [2].

\section{B. Power system model}

1) AC system model: We consider $N_{G}$ generating units represented by synchronous generators. To highlight the performance of the proposed control scheme, the dynamics of the excitation systems is not considered. Hence, every generator is modeled as a constant voltage source behind a direct axis transient reactance assuming constant flux linkages, as in [14], [15] for example. In addition, governor's actions are neglected and the mechanical power input to each machine is assumed to be constant during the transient period. Note that this assumption may be too restrictive if generators are equipped with fast-valving devices. Machines belonging to the same stations are assumed to be coherent and represented by a single equivalent machine.

Power system loads are represented as balanced constant admittances to neutral and the transmission lines are modeled by constant admittances, as in [16] for example.

2) HVDC system model: The representation of a HVDClink depends on the converter technology under consideration. In practice, two types of converters are concerned, namely current source converters and voltage source converters. The power electronics of current source converters is based on thyristors, which allow bidirectional active power flow but generally involve no control of reactive power injections at the terminal buses. This drawback is avoided with voltage source converters based on IGBT power electronics. Those converters may however induce other issues, regarding losses for example, which are discussed in [17].

In this paper, we will focus only on HVDC-links with current source converters, for which the quasi-steady state model proposed in [18] is chosen because it leads to reduced computation times with a limited loss in accuracy. Consequently, the HVDC-links' electrical dynamics is neglected with respect to the dynamics of the $\mathrm{AC}$ system, and the DC transmission line is modeled by a constant resistance. In addition, as recommended in [19], we assume that the reactive power injections of the current source converters at the AC terminal buses of an HVDC-link are determined by the corresponding active power injections.

As for the master control level, we make the assumption that the HVDC-links can respond to changes in their operation settings within less than 10 milliseconds. As of today, this assumption is probably too optimistic, as the dynamics of HVDC-links in terms of power tracking are of the order of 50 milliseconds (see [20] for example). Nevertheless, we motivate this assumption by the development of new technologies of converters that could lead to shorter time constants, and open new applications for fast-control of the HVDC settings.

During normal operation, the DC voltage is maintained constant at one terminal bus. When the current or bus voltages reach their minimum or maximum limits, the HVDC control mode is changed to maintain the concerned variable at its limit. In this case, one will observe variations of the power flow, even if the power flow setting is constant.

\section{Simulation results}

1) Illustrative example: For didactic purpose, this section presents simulation results obtained with the nine bus system for a three phase to ground fault on line $4-5$ at bus 5 . This fault is cleared by tripping line $4-5$ at time $t=250 \mathrm{~ms}$.

Simulations results obtained by operating the HVDC link with constant current are depicted in Figure 2, which represents the generators' relative phase angles with respect to the phase angle of generator 1 . It can be noticed that generators 2 and 3 accelerate more than generator 1 . The angular difference $\delta_{31}$ between generators 3 and 1 exceeds the stability limit defined as 180 degrees at time $t=528 \mathrm{~ms}$.

Figures 3 depicts the evolution of the generators' relative phase angles with the MPC controller using $D_{E}$ as transient stability index and a MPC control horizon equal to 3 . It shows that this controller prevents the system from instability, as the angular differences remain lower than 180 degrees. 


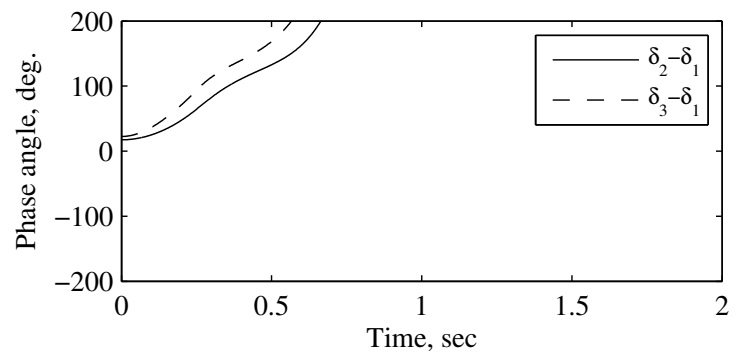

Fig. 2. Evolution of the relative voltage angles when a constant current setting is applied after a three phase to ground fault in the nine bus system on line 5-4 at bus 5 . The fault is cleared by tripping line $4-5$ at time $t=250$ ms.

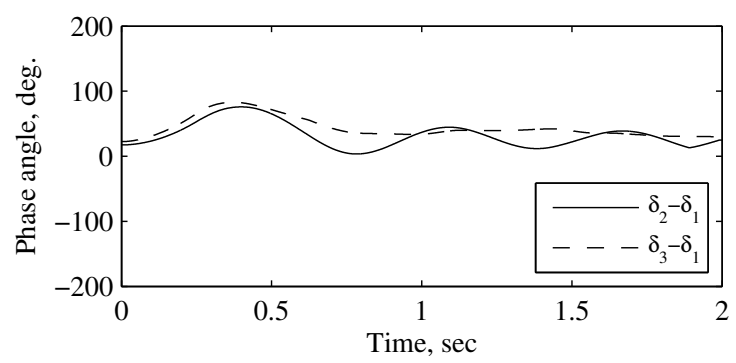

Fig. 3. Evolution of the relative voltage angles with the MPC scheme using index $D_{E}$ and $H=3$ after a three phase to ground fault in the nine bus system on line $5-4$ at bus 5 . The fault is cleared by tripping line $4-5$ at time $t=250 \mathrm{~ms}$.

2) Impact of the MPC control horizon $H$ : To analyze the influence of the control horizon $H$ on the effectiveness of the MPC control strategies, we present in Tables II and III the TTI values obtained with different values of $H$ for two faults on the 9 bus and 24 bus systems, respectively.

It can be observed that the TTI values tend to increase

TABLE II

TIME TO INSTABILITY (IN MS) OBTAINED WITH DIFFERENT VALUES OF THE MPC TIME HORIZON $H$ FOR A THREE PHASE TO GROUND FAULT AT BUS 3 ON THE LINE BETWEEN BUS 3 AND BUS 9 OF THE 9 BUS SYSTEM.

\begin{tabular}{|c|c|c|c|}
\hline \multirow{2}{*}{$H$} & \multicolumn{3}{|c|}{ Control strategy } \\
\cline { 2 - 4 } & $D_{P}$ & $D_{C}$ & $D_{E}$ \\
\hline \hline 1 & 343 & 353 & 350 \\
\hline 3 & 361 & 361 & 356 \\
\hline 5 & 361 & 361 & 360 \\
\hline 10 & 361 & 361 & 361 \\
\hline 15 & 361 & 361 & 361 \\
\hline
\end{tabular}

TABLE III

TIME TO INSTABILITY (IN MS) OBTAINED WITH DIFFERENT VALUES OF THE MPC TIME HORIZON $H$ FOR A THREE PHASE TO GROUND FAULT AT BUS 22 ON THE LINE BETWEEN BUS 21 AND BUS 22 OF THE 24 BUS SYSTEM.

\begin{tabular}{|c|c|c|c|}
\hline \multirow{2}{*}{$H$} & \multicolumn{3}{|c|}{ Control strategy } \\
\cline { 2 - 4 } & $D_{P}$ & $D_{C}$ & $D_{E}$ \\
\hline \hline 1 & 463 & 456 & 465 \\
\hline 3 & 477 & 475 & 475 \\
\hline 5 & 480 & 475 & 477 \\
\hline 10 & 480 & 477 & 479 \\
\hline 15 & 480 & 477 & 479 \\
\hline
\end{tabular}

with $H$, until they reach a limit value, which depends on the transient stability index. For the cases under consideration, the limit value is reached with $H=10$. However, we note that this limit value is generally well approximated with low values of $H$.

As the computation time is exponentially related to the control horizon, it is important to define $H$ as small as possible, while still ensuring good performance. Therefore, we have used $H=3$ in the simulations presented hereafter as a compromise between computation time and optimality of the solution.

3) Evaluation of the control strategies: Tables IV and V gather the TTI values obtained for different faults on the 9 bus and 24 bus systems, respectively. Different HVDC control strategies are compared, namely the $C C, P I, D_{P}, D_{C}, D_{E}$ and Optimal. Note that even with the Optimal strategy, the instability can not be avoided. We will therefore compare these strategies based only on their TTIs.

Although the Optimal control strategy considers a reduced space of control actions with respect to the PI strategy, the simulation results show that it improves significantly the TTIs when the fault is not located at a terminal bus. For example, with a fault at bus 1 on line $1-4$ of the 9 bus system, the TTI is increased by about $110 \%$ with the optimal solution. However, when the fault is located at a terminal bus, the actual power flow through the HVDC-link is zero regardless of the control strategy, and HVDC power flow modulation is of no additional value during the fault duration.

The simulation results also demonstrate that MPC with a limited time horizon of the three next time instants yields close to optimal performance, except for two particular cases (i.e., faults $4-5^{*}$ and $6^{*}-4$ on the 9 bus system), where the benefits of MPC are limited ${ }^{2}$. For all other cases, the stability indices $D_{P}, D_{C}$, and $D_{E}$ lead generally to similar types of actions and high values of the TTI. Nevertheless, none of the indices under consideration is best for all conditions.

It can also be observed that the PI controller yields only a small improvement with respect to a constant current setting $N C$. For example, the TTI improvement is limited to $13.0 \%$ for a fault at bus 1 on line $1-4$ of the 9 bus system. Such a low performance may be due to values of $K_{p}$ and $K_{i}$ that are not optimal for the cases under consideration. It may also come from the diversity of the fault locations. Indeed, as emphasized in [21], the PI controller inputs might not be sensitive enough to distant faults, and in this case, the benefits of PI control are small.

\section{CONCLUSIONS}

This paper presents a MPC strategy for HVDC power flow modulation to improve transient stability in power systems. At every instant, this discrete-time control scheme computes the control action by identifying an optimal sequence of successive control actions during a short time horizon. Early simulations

\footnotetext{
${ }^{2}$ Those particular cases are actually related to the relatively low value that has been chosen for $H$.
} 
TABLE IV

TIME TO INSTABILITY (IN MS) OBTAINED ON THE 9 BUS SYSTEM WITH DIFFERENT CONTROL STRATEGIES FOR DIFFERENT FAULT LOCATIONS. FAULT I-J* CORRESPONDS TO A THREE PHASE TO GROUND FAULT AT BUS J ON THE LINE BETWEEN BUS I AND BUS J.

\begin{tabular}{|c|c|c|c|c|c|c|}
\hline \multirow{2}{*}{ Fault } & \multicolumn{7}{|c|}{ Control strategy } \\
\cline { 2 - 7 } & $C C$ & $P I$ & $D_{P}$ & $D_{C}$ & $D_{E}$ & Optimal \\
\hline \hline $1^{*}-4$ & 330 & 373 & 693 & 693 & 691 & 693 \\
\hline $2^{*}-7$ & 357 & 364 & 426 & 430 & 427 & 437 \\
\hline $3^{*}-9$ & 205 & 206 & 361 & 361 & 356 & 361 \\
\hline $4^{*}-5$ & 299 & 299 & 299 & 299 & 299 & 299 \\
\hline $4-5 *$ & 392 & 468 & 448 & 754 & 748 & 754 \\
\hline $6^{*}-4$ & 309 & 340 & 850 & 358 & 824 & 856 \\
\hline $7^{*}-8$ & 288 & 312 & 445 & 448 & 442 & 448 \\
\hline $7-8 *$ & 260 & 271 & 799 & 798 & 807 & 812 \\
\hline $6-9 *$ & 203 & 203 & 203 & 203 & 203 & 203 \\
\hline
\end{tabular}

TABLE V

TIME TO INSTABILITY (IN MS) OBTAINED ON THE 24 BUS SYSTEM WITH DIFFERENT CONTROL STRATEGIES FOR DIFFERENT FAULT LOCATIONS.

\begin{tabular}{|c|c|c|c|c|c|c|}
\hline \multirow{2}{*}{ Fault } & \multicolumn{6}{|c|}{ Control strategy } \\
\cline { 2 - 7 } & $C C$ & $P I$ & $D_{P}$ & $D_{C}$ & $D_{E}$ & Optimal \\
\hline \hline $21-22^{*}$ & 321 & 323 & 477 & 475 & 475 & 482 \\
\hline $15-21^{*}$ & 361 & 361 & 361 & 361 & 361 & 361 \\
\hline $15^{*}-21$ & 476 & 498 & 752 & 758 & 754 & 788 \\
\hline $17-18^{*}$ & 379 & 380 & 809 & 782 & 783 & 809 \\
\hline $13^{*}-23$ & 443 & 488 & 460 & 463 & 447 & 468 \\
\hline $16^{*}-17$ & 477 & 504 & 701 & 713 & 709 & 713 \\
\hline $17^{*}-18$ & 462 & 483 & 866 & 860 & 847 & 868 \\
\hline
\end{tabular}

show that a time horizon of a few tens of milliseconds could be a good compromise between the performance of the scheme and the computational burden it involves. In addition, different cost functions based on common transient stability indices are evaluated on two benchmark systems with one embedded HVDC-link. Our proposed control strategy has also been compared with several other strategies for operating HVDC links in the aftermath of a disturbance (PI control based strategies, optimal strategy and constant settings for the active power flows). The simulation results show that using a MPC approach of HVDC modulation with real-time wide-area information as input is effective and can strongly improve time to instability for critical situations. Furthermore, the simulation results highlight the potential benefits of operating HVDClinks with transient overload margins in terms of time to instability.

Nevertheless, before applying this control strategy in real power systems, important issues have to be addressed. First, the empirical studies whose results have been reported in this paper have been carried by simplifying significantly the dynamics of a real power system. In particular time-delay issues, dynamics of the converters, and detailed generator models have not been taken into account. Second, in our simulations, we have assumed that the state of the system is fully observable and that an accurate model of the system is used by the MPC controller, which are quite restrictive assumptions. Finally, the time needed at every time step to solve of the optimisation problem by the MPC controller should not be neglected as we have done in our simulations. We believe that two promising research directions for addressing these different issues would be to design reduced models of the power system that will still be accurate for the control task at hand and investigate the use of more sophisticated techniques for solving rapidly the optimisation problems at the core of MPC techniques, even at the price of losing strict optimality.

\section{ACKNOWLEDGEMENTS}

Damien Ernst is a Research Associate of the Belgian FNRS, from which he acknowledges the financial support.

\section{REFERENCES}

[1] A. Hammad, J. Gagnon, and D. McCallum, "Improving the dynamic performance of a complex AC/DC system by HVDC control modifications," IEEE Transactions on Power Delivery, vol. 5, pp. 1934-1943, November 1990.

[2] G. Huang and V. Krishnaswamy, "HVDC controls for power system stability," in Proc. of the IEEE PES SM, vol. 1, pp. 597-602, July 2002.

[3] R. Eriksson, V. Knazkins, and L. Soder, "On the assessment of the impact of a conventional HVDC on a test power system," in Proc. of the IREP Symposium VII, August 2007.

[4] IEEE/CIGRE Joint Task Force on Stability Terms and Definitions, "Definition and classification of power system stability," IEEE Transactions on Power Systems, vol. 19, pp. 1387-1401, May 2004.

[5] C. Garcia, D. Prett, and M. Morari, "Model predictive control: Theory and practice - a survey," Automatica, vol. 25, no. 3, pp. 335-348, 1989.

[6] D. Ernst, M. Glavic, F. Capitanescu, and L. Wehenkel, "Reinforcement learning versus model predictive control: a comparison on a power system problem," IEEE Transactions on Systems, Man, and Cybernetics - Part B: Cybernetics, vol. 39, pp. 517-529, April 2009.

[7] M. Larsson, D. Hill, and G. Olsson, "Emergency voltage control using search and predictive control," International Journal of Power and Energy Systems, vol. 24, no. 2, pp. 121-130, 2002.

[8] B. Otomega, A. Marinakis, M. Glavic, and T. Van Cutsem, "Model predictive control to alleviate thermal overloads," IEEE Transactions on Power Systems, vol. 22, pp. 1384-1385, August 2007.

[9] J. Ford, G. Ledwich, and Z. Dong, "Efficient and robust model predictive control for first swing transient stability of power systems using flexible ac transmission systems devices," IET Proceedings on Generation, Transmission, and Distribution, vol. 2, no. 5, pp. 731-742, 2008.

[10] J. Pearl, Heuristics: Intelligent Search Strategies for Computer Problem Solving. Addison-Wesley, 1984.

[11] P. Hart, N. Nilsson, and R. Bertram, "A formal basis for the heurisitc determination of minimum cost paths," IEEE Transactions on Systems Science and Cybernetics, vol. 4, pp. 100-107, July 1968.

[12] C. Fu and A. Bose, "Contingency ranking based on severity indices in dynamic security analysis," IEEE Transactions on Power Systems, vol. 14, pp. 980-986, August 1999.

[13] IEEE Reliability Test System Task Force of Applications of Probability Methods Subcommittee, "IEEE reliability test system-96," IEEE Transactions on Power Systems, vol. 14, pp. 1010-1020, August 1999.

[14] M. Pai, Energy Function Analysis for Power System Stability. Kluwer Academic Publishers Group, 1989.

[15] M. Pavella and P. Murthy, Transient Stability of Power Systems: Theory and Practice. Wiley, 1993.

[16] P. Anderson and A. Fouad, Power System Control and Stability, vol. 1. The Iowa State University Press, 1977.

[17] J. Arrillaga, N. Watson, and Y. Liu, Flexible Power Transmission: The HVDC Options. Wiley \& Sons, 2007.

[18] B. Johnson, "HVDC models used in stability studies," IEEE Transactions on Power Delivery, vol. 4, pp. 1153-1163, April 1989.

[19] P. Kundur, Power System Stability and Control. McGraw-Hill, 1994.

[20] IEEE AC/DC System Dynamics Task Force, "Dynamic performance characteristics of north-american hvdc systems for transient and dynamic stability evaluations," IEEE Transactions on Power Apparatus and Systems, vol. 100, pp. 3356-3364, July 1981.

[21] J. Hazra, Y. Phulpin, and D. Ernst, "HVDC control strategies to improve transient stability in interconnected power systems," in Proc. of the IEEE PowerTech, pp. 1-6, June 2009. 